\title{
Documentation pour un projet de conception
}

\author{
François Charron, ing. \\ Département de génie mécanique \\ Université de Sherbrooke \\ Francois.R.Charron@USherbrooke.ca
}

\author{
Jean-Philippe Desbiens, ing. jr. \\ Département de génie mécanique \\ Université de Sherbrooke \\ Jean-Philippe.Desbiens@USherbrooke.ca
}

\section{Résumé}

Le Département de génie mécanique de l'Université de Sherbrooke a introduit des projets majeurs de conception dans son programme de baccalauréat depuis 1992. Évidemment, un des objectifs des projets de conception est de permettre aux étudiants de développer une compétence en rédaction de rapports techniques portant sur la conception. Jusqu'à ce jour, aucun document de référence, soit l'équivalent d'un livre de référence dans un cours magistral, était disponible pour guider les étudiants dans la rédaction de leurs rapports de conception. Afin de combler ce besoin, un projet majeur de conception fut donc réalisé et une documentation complète de référence a été développée.

\section{Introduction}

La formation d'ingénieur intègre deux éléments indissociables et complémentaires qui sont le génie et l'ingénierie. Le génie englobe le corpus des connaissances et des techniques de l'ingénieur, et l'ingénierie représente l'étude complète d'un projet industriel sous toutes ses facettes (techniques, économiques, sociales, environnementales, etc.).

Évidemment, la formation d'un ingénieur compétent doit nécessairement intégrer ces deux éléments. Dans tous les programmes de baccalauréat en génie au Canada, on retrouve certainement une formation adéquate en génie (par exemple pour le génie mécanique, une formation portant sur les matériaux, la mécanique des solides, la mécanique des fluides, la thermodynamique, etc.). Par contre, la formation en ingénierie est souvent déficiente. Les étudiants ont relativement peu de formation sur les processus et les méthodologies utilisés par l'ingénieur lors du développement et de la conception de produits, de procédés, de machines, de systèmes, etc. De plus, lors de leur formation, les étudiants ont relativement peu d'occasions de développer des compétences ${ }^{1}$ techniques et professionnelles par l'entremise de projets de conception.

Au cours de la réforme du programme de baccalauréat en génie mécanique (1992 à 1996), le Département a beaucoup réfléchi à la question du développement des compétences techniques et professionnelles chez les étudiants. À la suite d'un constat concernant la pertinence d'une approche de formation par compétence pour la formation en génie, le Département a retenu un certain nombre de compétences et il s'est interrogé sur les approches pédagogiques nécessaires pour le développement de celles-ci. Cette réflexion sur le développement de compétences a convaincu le Département qu'il était nécessaire de mettre en place des projets d'intégration (mini projet de conception) au cours des quatre premières sessions d'étude du programme et un projet majeur de conception pour les quatre dernières sessions.

La mise en place des projets majeurs de conception a donc nécessité le développement d'une nouvelle approche pédagogique, de nouveaux laboratoires, de nouveaux outils d'évaluation, etc. [1].

1 La compétence combine des savoirs, des savoir-faire et des savoir-être qui permettent à une personne de prendre des actions précises dans le cadre de son travail. Le développement des compétences auprès des étudiants nécessitent un environnement d'apprentissage permettant à l'apprenant d'intégrer des savoirs (savoir comprendre, savoir interpréter, savoir agir sur mesure, etc.), des savoirfaire (savoir coopérer, savoir raisonner, savoir procéder, savoir apprendre, etc.) et des savoir-être (ex. savoir se comporter, etc.), d'assurer un rôle de responsabilité par rapport à son apprentissage, de démontrer sa capacité de résoudre des problèmes ouverts et mal définis, et d'être capable de mettre en pratique ses acquis dans un nouveau contexte (re-contextualisation). 
Un des principaux résultats obtenus lors des projets majeurs de conception est la remise de rapports de développement de produits (ou de machines, des systèmes, etc.) à la fin de chacune des sessions. Or, contrairement à la leçon magistrale où il existe une quantité importante de livres de référence dans presque tous les domaines du génie, malheureusement, il n'existe pas de rapports de conception dans la littérature que les étudiants pourraient consulter comme référence.

À la suite de ce constat, il a été convenu de réaliser un projet de conception complet au Département de génie mécanique et de rédiger toute la documentation technique associée à chacune des étapes du processus de développement de produit (PDP). Cette documentation deviendrait alors une référence pour les étudiants lors de l'élaboration des rapports de conception.

La présente publication est principalement divisée en deux parties. La première discutera du projet de conception « $\mathrm{McGrO}$ » réalisé au Département et par la suite, la documentation développée sera présentée.

\section{Le projet de conception « McGrO»}

\subsection{Contexte}

Dans le but de maximiser la portée des résultats du projet « $\mathrm{McGrO}$ », il a été décidé de développer une machine à commande numérique à trois axes permettant l'usinage de moules pour le thermoformage de plastiques ou la mise en forme de matériaux composites. Une telle machine n'existait pas au Département mais il y avait un besoin important pour plusieurs projets de conception réalisés par les étudiants.

La documentation technique du projet « $\mathrm{McGrO}$ » fut donc rédigée à la fin de chacune des principales étapes de la conception en simulant les mêmes activités exigées aux étudiants lors de la réalisation de leurs projets de conception. Il est évident qu'un rapport de conception présente les principaux résultats obtenus lors de la session.

La Figure 1 illustre le processus de développement de produit (PDP) développé pour les projets de conception faits par les étudiants et utilisé dans le cadre du projet « $\mathrm{McGrO} »$.

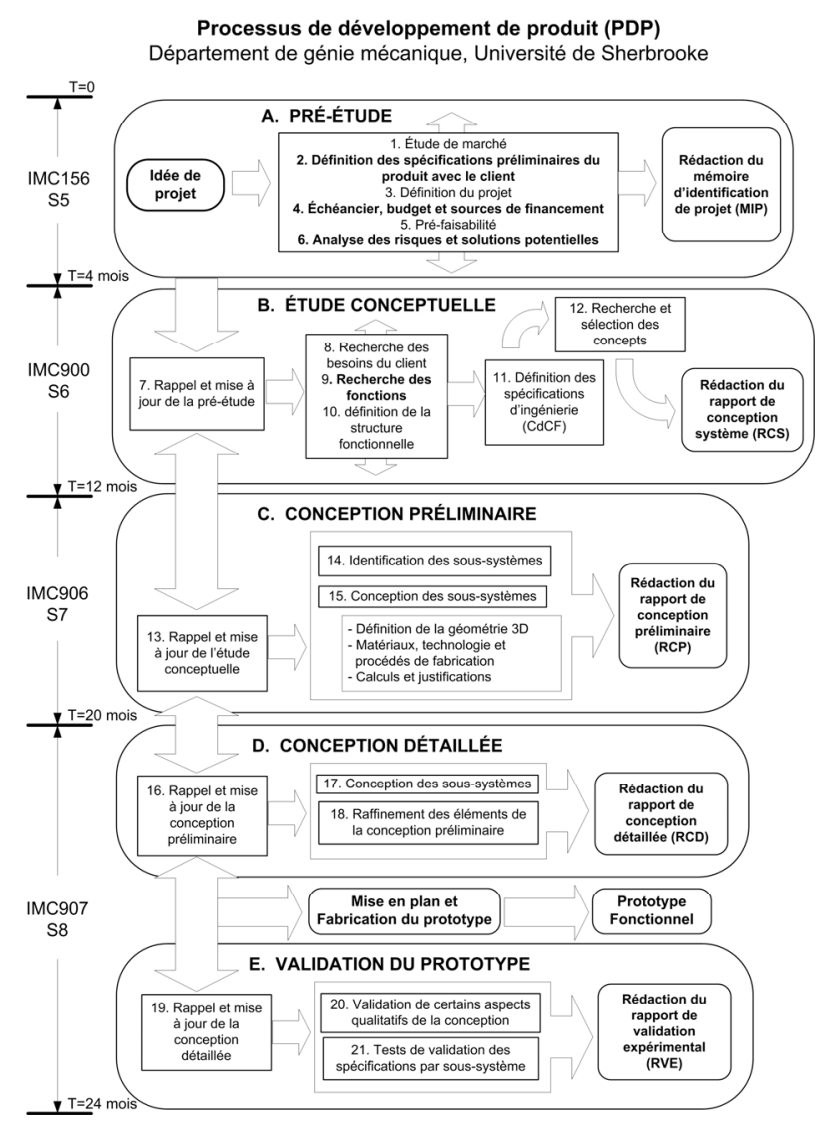
Figure 1. Processus de développement de
produit (PDP)

Les grandes étapes du PDP sont les suivantes :

1. Pré-étude

2. Étude conceptuelle

3. Conception préliminaire

4. Conception détaillée

5. Fabrication et validation du prototype

Pour chacune des cinq (5) grandes phases du PDP, un rapport synthèse doit être rédigé par les étudiants. Il est important de savoir que ces rapports sont écrits avec l'aide d'un journal de bord (logbook). Chacun des membres de l'équipe doit documenter l'ensemble de son travail (ex. ses réflexions, ses calculs, ses justifications, ses contacts, etc.). Cet outil est d'ailleurs imposé aux étudiants pendant leurs projets de conception et il est utilisé pour l'évaluation du travail individuel de chacun des membres d'une équipe.

Finalement, il est important de remarquer que deux contraintes majeures avaient été imposées au projet « $\mathrm{McGrO} »$ dès la pré-étude, soit :

- Une durée de 4 mois pour réaliser le projet 
- Un budget de $10000 \$$ alloué pour la fabrication du prototype

Tout au long du projet, ces contraintes ont été considérées au même titre que les autres besoins de nos clients.

\subsection{Réalisation du projet « McGrO »}

Tel que mentionné préalablement, le mandat du projet $\mathrm{McGrO}$ était de concevoir un équipement permettant de réaliser des moules en trois dimensions pour effectuer la mise en forme de polymères et de matériaux composites.

\subsubsection{Formation de l'équipe de projet}

Pour réaliser le projet « $\mathrm{McGrO}$ » dans le temps prévu, une équipe fut constituée. Les principales personnes impliquées furent les suivantes :

- 4 professeurs (méthodologie, documentation, supervision);

- 1 assistant de recherche en conception (méthodologie, conception, fabrication);

- 2 stagiaires au niveau du baccalauréat (mécatronique, conception, fabrication);

- 3 techniciens en fabrication mécanique (fabrication);

- 3 techniciens en mécatronique/informatique (aspects mécatroniques).

\subsubsection{Conception}

Dès janvier 2002, la conception de la machine « $\mathrm{McGrO}$ » a débuté par une pré-étude complète des technologies existantes dans le domaine des machinesoutils à commande numérique à trois axes (3D) et de la fabrication de moule fait de matériaux mous (ex. bois et mousses synthétiques).

Les étapes du PDP présenté dans la Figure 1 ont été suivies par l'équipe de projet. Évidemment, ce PDP s'inspire fortement de processus modernes de développement de produits et, par conséquent, plusieurs activités du PDP sont généralement réalisées d'une façon simultanée.

\subsubsection{Fabrication}

La fabrication du prototype débuta dès que la conception de quelques éléments simples de la structure principale fut terminée. Pendant ce temps, des calculs avancés concernant les éléments mécaniques plus complexes ont été faits.
Afin de limiter les délais dans la fabrication, des pièces mécaniques sélectionnées en raison de certaines contraintes furent commandées chez les fournisseurs avant la fin de la conception détaillée. La figure suivante présente le prototype final.

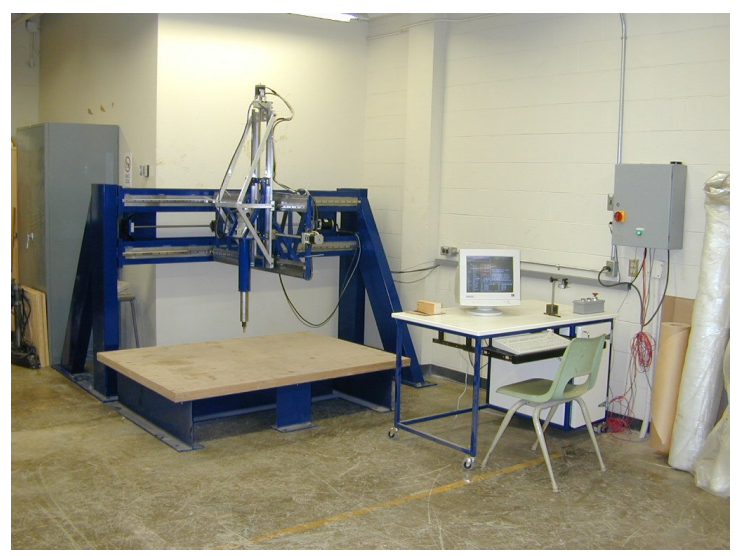

Figure 2. Machine à commande numérique 3 axes « McGrO »

\subsubsection{Validation expérimentale}

La phase de validation expérimentale consista à tester le fonctionnement du prototype en s'assurant qu'il respecte les attentes des clients ainsi que les spécifications d'ingénierie définies dans le cahier des charges fonctionnel (CdCF) créé lors de la phase de conception système (RCS). Pour ce faire, différents tests ont été effectués au niveau des sous-systèmes et de leurs composantes afin d'évaluer les performances.

La phase de validation du prototype « $\mathrm{McGrO}$ » a mis en évidence certaines lacunes de conception (ex. problème de positionnement dans un axe) et certaines modifications mécaniques ont dues être effectuées pour satisfaire les exigences du $\mathrm{CdCF}$.

\section{Documentation}

\subsection{Définition des documents}

Les documents rédigés lors du projet « $\mathrm{McGrO}$ » sont les suivants :

- Mémoire d'identification de projet (MIP)

- Rapport de conception système (RCS)

- Rapport de conception préliminaire (RCP)

- Rapport de conception détaillée (RCD)

- Rapport de validation expérimentale (RVE) 
Dans les grandes lignes, un rapport est rédigé à la fin de chacune des quatre sessions (voir la figure 1) à l'exception de la dernière session où deux rapports sont écrits (S5 - MIP, S6 - RCS, S7 - RCP et S8 $\mathrm{RCD}$ et RVE). De plus, les équipes doivent faire une présentation orale des résultats de chacun des rapports (revue de conception). Cette présentation orale permet aux étudiants d'apprendre à communiquer efficacement des résultats de conception mais aussi de découvrir l'avancement des travaux de leurs consoeurs et confrères.

Les prochaines sections présentent les cinq rapports.

\subsection{Mémoire d'identification de projet (MIP)}

La pré-étude permet d'établir les informations essentielles à partir desquelles le projet pourra être élaboré plus en détails. Le mémoire d'identification de projet est le rapport qui permet de présenter les résultats de cette pré-étude de faisabilité du projet.

Lors de cette phase, les spécifications préliminaires sont élaborées (dans certains cas, avec le client) afin de mieux cerner les performances attendues du produit, du système ou de la machine. Les contraintes (ex. budget maximum) sont également identifiées. Par la suite, l'équipe de conception effectue une revue de la littérature et une pré-étude de marché pour vérifier si le mandat du projet est réalisable compte tenu des contraintes et des spécifications préliminaires. Le tout est documenté et au besoin, quelques calculs préliminaires ou validations simples sont réalisées pour vérifier la faisabilité du projet.

Finalement, le MIP est rédigé pour exposer les principaux résultats de la pré-étude et pour définir le projet d'une façon claire. Pour illustrer le contenu du MIP, voici la table des matières pour le projet « $\mathrm{McGrO} »$ :

1 Introduction

2 Nature du projet

2.1 Description

2.2 Produits similaires

2.3 Problématique

2.4 Objectifs du projet

3 Formulation préliminaire du projet

3.1 Principales spécifications

3.2 Principales contraintes

3.3 Livrables

3.4 Échéancier

4 Ressources

4.1 Description de l'équipe

4.1.1 Fabrication et essais

4.2 Budget et sources de financement

5 Études complémentaires

6 Gestion des risques
7 Recommandations

8 Approbation

Annexe I : Étude de préfaisabilité de marché et des technologies commerciales

Annexe II : Étude de préfaisabilité technique

Annexe III : Étude de préfaisabilité de l'usinage du MDF et de la mousse de polystyrène

Annexe IV : Étude de préfaisabilité économique Références

Afin d'illustrer concrètement le travail de rédaction, des extraits du MIP sont fournis dans l'annexe A. Selon les conclusions du MIP, le projet est mis de l'avant ou la définition du projet est modifiée en accord avec le client. À noter que cette phase permet d'identifier des solutions techniques potentielles ou restrictives (contraintes) qui simplifient les choix de conception lors du PDP en imposant des concepts pour certains sous-systèmes dès le départ (ex. : choix d'un logiciel de FAO respectant le budget lors de la préétude du projet « $\mathrm{McGrO} »)$.

\subsection{Rapport de conception système (RCS)}

Le rapport RCS présente les principaux résultats de l'ingénierie des systèmes. Lors de cette phase (voir la figure 1), les besoins du client sont identifiés, analysés et classés par affinité. Par la suite, les fonctions du produit sont identifiées avec l'aide de différentes techniques d'analyse (ex. méthode des interacteurs, FAST, etc.). Avec les besoins du client et les fonctions $\mathrm{du}$ produit, les spécifications fonctionnelles sont définies. Pour chacune des fonctions, le niveau, la flexibilité et l'importance ${ }^{2}$ sont fixés à la suite d'estimations ou grâce aux données techniques recueillies durant la pré-étude. Ces fonctions sont alors documentées dans un cahier des charges fonctionnel (CdCF).

Par la suite, la structure fonctionnelle du produit est élaborée pour déterminer les principaux sous-systèmes du produit. Cette structure fonctionnelle est définie à partir des principales fonctions d'usage du produit ( ex. : contrôler les axes, faire tourner l'outil, etc.).

La recherche de concepts peut ensuite être réalisée par différentes approches. Les concepts doivent permettre de concrétiser les principales fonctions d'usage des sous-systèmes. Par la suite, les concepts sont évalués en utilisant les critères reposant directement sur les besoins du client ou plus spécifiquement, dans certains cas, les principales spécifications fonctionnelles du CdCF.

2 Le déploiement de la fonction qualité (DFQ 1) est également une technique utilisée pour pondérer les spécifications fonctionnelles (et les spécifications techniques dans certains cas). 
Finalement, le rapport de RCS inclut le choix des concepts finaux pour le produit et chacun des soussystèmes. Ces concepts seront détaillés dans les étapes de conception subséquentes.

La table des matières du rapport de RCS est la suivante :

\section{Introduction}

2 Rappel et mise à jour

2.1 Résultats des études complémentaires réalisées après le MIP

2.2 Nature du projet

2.3 Formulation préliminaire du projet

2.4 Ressources

2.5 Gestion des risques

3 Analyse des besoins

3.1 Clients

3.2 Identification des besoins

3.3 Analyse et classement des besoins

4 Analyse fonctionnelle

4.1 Recherche des fonctions

4.2 Création de l'arbre fonctionnel

5 Spécifications

5.1 Spécifications fonctionnelles

5.2 Spécifications de contrainte

5.3 Pondération DFQ-1

6 Configuration

6.1 Structure fonctionnelle

6.2 Configuration physique

7 Émergence et sélection des concepts

7.1 Concepts dictés par les contraintes, les orientations techniques et la structure fonctionnelle

7.2 Génération des concepts

7.3 Concepts retenus pour chaque sous-système

7.4 Révision de la configuration

8 Suite des travaux

8.1 Mise à jour du budget et des sources de financement

8.2 Mise à jour de l'échéancier

Annexe I : Résultats des études complémentaires

Annexe II : Mise à jour du budget

Annexe III : Liste brute des besoins

Annexe IV : Liste des fonctions

Annexe V : Cahier des charges fonctionnel

Annexe VI : Matrice DFQ-1

Annexe VII: Cahier des concepts et méthode de Pugh

Références

On constate que le rapport RCS, ainsi que les rapports subséquents, incluent toujours une mise à jour des principaux résultats des étapes précédentes du PDP ainsi qu'un suivi de l'échéancier et du budget. Des extraits du rapport de RCS sont inclus dans l'annexe B.

\subsection{Rapports de conception préliminaire (RCP) et détaillée (RCD)}

Lors de la phase de conception préliminaire, le dimensionnement du produit est amorcé. Dans un premier temps, la modélisation géométrique des concepts est faite avec l'aide d'un logiciel de conception assistée par ordinateur (CAO). Cette modélisation est développée à partir de la structure fonctionnelle, de la configuration physique ainsi que des schémas des concepts.

Les technologies, les matériaux, les composantes, etc. sont choisis en fonction des concepts retenus, des performances spécifiées dans le $\mathrm{CdCF}$ et des contraintes du projet (ex. : temps, budget, ressources humaines, etc.). Des justifications suffisantes viennent appuyer les choix de conception. Ces dernières peuvent prendre la forme de calculs analytiques, d'explications reposant sur des analogies, de simulations numériques, etc. Évidemment, si les performances envisagées du produit et/ou des soussystèmes ne sont pas rencontrées, des modifications aux concepts sont proposées et/ou même, une mise à jour du CdCF peut être effectuée.

Après la phase de RCP, la phase de conception détaillée est progressivement mise en route. Les activités de la conception détaillée permettent de préciser et de mettre à jour les principaux résultats de la conception préliminaire (ex.: modélisations, calculs, justifications, dessins techniques, procédés de fabrication, etc.).

La table des matières du rapport de RCP/RCD est la suivante :

1 Introduction

2 Rappel et mise à jour

2.1. Activités faites depuis la RCS

2.2. MIP

2.2.1. Nature du projet

2.2.2. Formulation finale du projet

2.2.3. Ressources

2.2.4. Gestion des risques

2.3. Conception système

2.3.1. Analyse des besoins

2.3.2. Analyse fonctionnelle

2.3.3. Spécifications

2.3.4. Configuration

2.3.5. Émergence et sélection des concepts

2.4. Révision de la configuration

3 Conception du système « McGrO »

3.1. Définition de la marge de sécurité

3.2. Calculs préliminaires

4 Conception du sous-système châssis

4.1. Partie $n$ du sous-système châssis $(n=1,2$, etc.)

4.1.1. Géométrie 3D

4.1.2. Matériaux, techniques et fabrication

4.1.3. Calculs et justifications 
5 Conception du sous-système déplacement linéaire

5.1. Partie $n$ du sous-système déplacement linéaire $(n=1,2$, etc. $)$

5.1.1. Géométrie 3D

5.1.2. Matériaux, techniques et fabrication

5.1.3. Calculs et justifications

6 Conception du sous-système de commande

6.1. Partie $n$ du sous-système de commande $(n=1,2$, etc.)

6.1.1. Géométrie 3D

6.1.2. Matériaux, techniques et fabrication

6.1.3. Calculs et justifications

\section{Suite des travaux}

7.1. Mise à jour du budget et des sources de financement

7.2. Mise à jour de l'échéancier

Annexe I : Mise à jour du budget du projet " McGrO »

Annexe II : Mise à jour du CdCF

Annexe III : Mises en plan du projet « McGrO»

Annexe IV : Détails des calculs pour la validation du châssis mobile de l'axe $X$

Annexe V : Schéma de câblage électrique de «McGrO»

Références

Évidemment, puisque les activités des conceptions préliminaire et détaillée sont assez analogues, les contenus des rapports sont très similaires à l'exception du niveau de détails des résultats (ex. : modélisation, calculs, etc.). Des extraits du rapport de RCD sont inclus à l'annexe $\mathrm{C}$.

\subsection{Rapport de validation expérimentale du prototype (RVE)}

La conformité du produit conçu est présentée dans le rapport de validation expérimentale du prototype. Pour ce faire, le cahier des charges fonctionnel est repris et une liste des principales spécifications techniques pouvant être validées considérant les contraintes techniques, le budget et l'échéancier est préparée.

Par exemple pour le projet « $\mathrm{McGrO} »$, plusieurs spécifications définissant la précision d'usinage ont été validées (voir le Tableau 1). Ces spécifications ont été incluses dans la liste des spécifications pour la validation expérimentale.

Tableau 1 - Extrait des spécifications techniques liées à la précision d'usinage

\begin{tabular}{|c|c|c|}
\hline Spécifications techniques & Niveau & Flexibilité \\
\hline $\begin{array}{c}\text { Précision de la position de } \\
\text { l'origine de la pièce }\end{array}$ & $2 \mathrm{~mm}$ & Max. \\
\hline $\begin{array}{c}\text { Angle d'usinage vertical } \\
\text { minimal }\end{array}$ & $15^{\circ}$ & $\pm 2^{\circ}$ \\
\hline $\begin{array}{c}\text { Précision d'usinage des } \\
\text { surfaces }\end{array}$ & $5 \mathrm{~mm}$ & $\pm 1 \mathrm{~mm}$ \\
\hline $\begin{array}{c}\text { Précision de la position de } \\
\text { l'outil }\end{array}$ & $0,80 \mathrm{~mm}$ & 0 \\
\hline
\end{tabular}

Les spécifications qui ne nécessitent pas de mesures expérimentales pour la validation sont également identifiées et validées.

Par la suite, des tests expérimentaux sont élaborés pour les sous-systèmes et la machine. Les divers tests expérimentaux sont menés et les résultats sont documentés dans le RVE. L'ensemble des résultats de validation permet de savoir si les performances du produit respectent les spécifications définies dans le CdCF. Si les spécifications techniques ne sont pas respectées, le prototype est modifié, si possible, afin d'améliorer la conception.

La table des matières du rapport de validation expérimentale (RVE) est la suivante :

1 Introduction

2 Rappel et mise en contexte

2.1 Nature du projet

2.2 Besoins, fonctions et spécifications

2.3 Conception et intégration

2.3.1 Changements de conception

3 Approche de validation

4 Plan de validation des aspects qualitatifs de la conception

5 Plan de validation expérimental au niveau des sous-systèmes

5.1 Test 1 - sous-système de déplacement linéaire

5.1.1 Objectifs

5.1.2 Méthodologie (montage, instrumentation, etc.)

5.1.3 Résultats

5.1.4 Discussion

5.2 Test $\mathrm{N}$ - sous-système « $\mathrm{X}$ »

6 Plan de validation expérimental pour « McGrO»

6.1 Objectifs

6.2 Méthodologie (montage, instrumentation, etc.)

6.3 Résultats

6.4 Discussion

7 Retour sur la satisfaction du client

8 Synthèse et recommandations

8.1 Mise à jour du budget et des sources de financement

8.2 Mise à jour de l'échéancier

Annexe I : Mise à jour du budget du projet «McGrO »

Annexe II : Cahier des charges final

Annexe III : Machine CNC « McGrO»-guide de l'utilisateur

\section{Références}

Des extraits du rapport de RVE sont inclus à l'annexe D.

\section{Résultats secondaires}

Il est important de mentionner qu'en plus de la réalisation du prototype de la machine « $\mathrm{McGrO}$ » ainsi que des documents techniques de référence du 
PDP, d'autres résultats secondaires sont issus du projet « $\mathrm{McGrO} »$.

Entre autres, une lacune avait été identifiée dans les compétences des étudiants en dessin technique, particulièrement au niveau de la réalisation de dessins de détails et d'assemblage. Dans le but de fournir une aide concrète aux étudiants, les mises en plan du projet « $\mathrm{McGrO}$ » ont été réalisées consciencieusement en prenant soin de respecter les normes du dessin technique et de couvrir la majorité des cas de cotation rencontrés typiquement par les étudiants de génie mécanique. Des exemples concernant les mises en plan réalisées avec le logiciel de CAO SolidWorks sont donc maintenant accessibles aux étudiants via le site Internet de conception du Département de génie mécanique (www.conception.gme.usherb.ca).

Également, nous avons choisi d'acheter un logiciel de fabrication assistée par ordinateur $(\mathrm{FAO})^{3}$ pour générer les trajectoires de coupe de la machine à commande numérique « $\mathrm{McGrO} »$. L'achat de ce logiciel permettra donc aux étudiants utilisant la machine « $\mathrm{McGrO}$ » de bénéficier d'une courte formation en FAO et d'utiliser un logiciel couramment utilisé en fabrication automatisée par ordinateur. Un manuel d'utilisateur a d'ailleurs été rédigé pour initier les étudiants à l'utilisation proprement dite de la machine « $\mathrm{McGrO}$ » ainsi qu'aux concepts généraux de la FAO.

Il est à noter également que la machine « $\mathrm{McGrO} » \mathrm{a}$ permis la réalisation de plusieurs moules en bois pour des projets de conception réalisés à l'automne 2003. La Figure 3 montre un moule en contreplaqué pour le moulage d'un traîneau de sauvetage en fibre de verre réalisé pour la station de ski Sutton des Cantons de l'est.

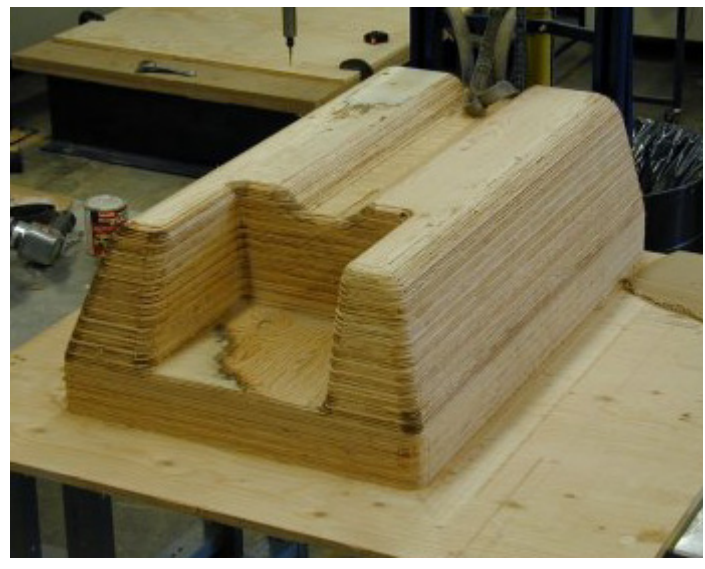

Figure 3. Moule usiné du projet Yéti

\footnotetext{
${ }^{3}$ FeatureCAM
}

\section{Publication}

Tous les documents produits lors du projet « $\mathrm{McGrO}$ » sont désormais en ligne sur le site Internet de la conception du Département de génie mécanique au www.conception.gme.usherb.ca. La section «Étude de cas » du site Internet est entièrement dédiée au projet « McGrO » ainsi qu'à la présentation des principaux résultats. On retrouve dans cette section :

- Tous les rapports techniques de conception (MIP, RCS, RCP, RCD et RVE) ainsi que leurs annexes en format PDF;

- Le manuel d'utilisation de «McGrO» ainsi que des exemples de moules fabriqués avec la machine à commande numérique;

- Les tutoriaux du logiciel FeatureCAM pour la fabrication assistée par ordinateur des moules;

- Des outils de rédaction pour les rapports techniques du PDP;

- Des exemples de mises en plan d'assemblage et de détails réalisés avec le logiciel de CAO SolidWorks 2003 faits selon les règles de l'art du dessin technique.

Bref, les étudiants du Département du génie de la conception mécanique disposent désormais d'outils uniques pour les aider dans la réalisation de leur projet de conception.

\section{Conclusion}

L'objectif principal du projet «McGrO» était de documenter un projet de conception réalisé selon les étapes du processus de développement de produits du Département de génie mécanique. Les documents techniques du projet « McGrO » ont été créés, ils sont en ligne et ils peuvent être consultés par tous les étudiants afin de les aider dans la rédaction technique de leurs rapports de projet de conception.

De plus, la documentation du projet « $\mathrm{McGrO}$ » servira également à l'élaboration d'études de cas présentées dans le cours de méthodologie de conception enseigné en S5. Ce cours est une introduction au PDP et, à la méthodologie et aux outils de conception. De plus, ce cours est utilisé pour amorcer les projets de conception (formation des équipes et réalisation du MIP).

Finalement, le Département est désormais muni d'une machine à commande numérique pour aider les étudiants dans la fabrication de moules pour la mise en forme de polymères et de matériaux composites. D'ailleurs, la machine est en service depuis quelques 
mois et elle a été utilisée à plusieurs reprises avec succès.

\section{RÉFÉRENCES}

[1] Charron, F. et coll., Design Projects in the Mechanical Engineering Curriculum at Sherbrooke University Past, Present and Future, 2002 ASEE Annual Conference, Montréal, June 2002.

\section{ANNEXE A - EXTRAITS DU MIP}

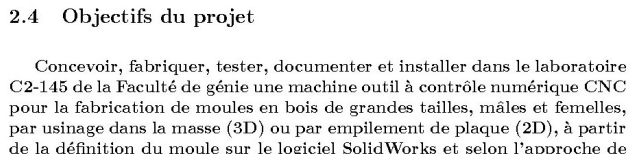
C2-145 de la Faculté de génie une machine outil à contrôle numérique CNC pour la fabrication de moules en bois de grandes tailles, máles et femelles,

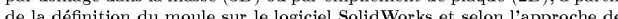

3 Formulation préliminaire du projet

3.1 Principales spécifications

Les études de pré-faisabilité des annexes I à IV ont permis d'établir de façon préliminaire certaines specifications d'ingenierie globales. Celles-ci ont été validées lors de rencontres avec le client. Elles sont établies afin d'orienter et d'accélerer le dércloppement de $\mathrm{McGr}$

1. Taille maximale des moules

- Définition : Dimensions hors tout du plus grand moule à fabriquer.

- Niveau : $2.50 \mathrm{~m} \times 1.25 \mathrm{~m}$, hauteur $1.00 \mathrm{~m}$

- Flexibilité : $-0.10 \mathrm{~m}$ sur chaque dimension

Les grands moules sont usinés par partie puis assemblés. On limite à trois le nombre de parties a assembler pour obtenir le moule de $2.50 \mathrm{~m}$ de longueur. Les a do blo de bois a seulpter sont donc : $0.83 \mathrm{~m} \times 1$

2. Courses maximales

- Définition : Déplacements maximums de l'outil de la machine CNC

- Niveau :

(a) Course en longueur ${ }^{1}: 1.00 \mathrm{~m}$

(b) Course en largeur : $1.50 \mathrm{~m}$

(c) Course en hauteur : $1.00 \mathrm{~m}$

- Flexibilité : $-0.10 \mathrm{~m}$ sur chaque dimension

3. Taille typique d'un moule (ou d'une section de moule) de bois pour le calcul du temps d'usinage

${ }^{1}$ Les axes $X, Y$ et $Z$ ne sont pas encore assignaés à ce noment-ci

6 Gestion des risques

Les principaux risques identifiés à ce jour sont :

1. Manque de ressources humaines pour la fabrication;

2. Incompatibilité de SolidWorks pour l'interface CAO-McGrO;

3. Imprévus financiers.

L'évaluation comparative des risques est présentée au tableau 2. Il en ressort que le risque principal est le manque de ressources humaines pour la fabrication.

TABLEAU 2: Évaluation des risques

\begin{tabular}{l|c|c|c|c}
$\begin{array}{l}\text { Événement } \\
\text { critique }\end{array}$ & $\begin{array}{l}\text { Probabilité } \\
\text { d'occurence } \\
a \in[0-5]^{1}\end{array}$ & $\begin{array}{l}\text { Gravité de } \\
\text { limpact } \\
b \in[0-5]^{1}\end{array}$ & $\begin{array}{l}\text { Niveau de } \\
\text { risque } \\
c=a \times b\end{array}$ & Priorisation \\
\hline \hline $\begin{array}{l}\text { Manque de } \\
\text { ressourcess hu- } \\
\text { maines pour la } \\
\text { fabrication }\end{array}$ & 3 & 5 & 15 & 1 \\
\hline $\begin{array}{l}\text { Imprévus fi- } \\
\text { nanciers }\end{array}$ & 3 & 3 & 9 & 2 \\
$\begin{array}{l}\text { Incompatibilité de } \\
\text { SolidWorks }\end{array}$ & 2 & 4 & 8 & 3 \\
\hline
\end{tabular}

10 faible à 5 :elevé

Les mesures de mitigation potentielles des risques précédemment énumérés son par ordre de priorité de risque

1. Manque de ressources humaines pour la fabrication :

(a) devancer au maximum le début de la fabrication de façon à utiliser le maximum dieures avant le debut des vacances annterlas. Ceite mesure implique qu'une priorite soit donnée à la conception détaillée de la structure principale de la machine;

(b) faire fabriquer certaines pièces par des sous-traitants. Le coût estimé de la fabrication des principales pièces de la structure par des sous-traitant est de 50008 . Compte tent de la probabilité doccurence de $3 / 5=60 \%$, un montant de 3 000s est ajoute à lestimé budgetare du tableau 5 de

2. Incompatibilité de SolidWorks pour l'̈nterface CAO-McGrO : Advenant une trop grande difficulté à développer une interface avec le logiciel 


\section{ANNEXE B - EXTRAITS DU RCS}

\section{Analyse des besoins}

\subsection{Clients}

Le principal client du projet McGrO est le Département de génie mécanique. Ce dernier englobe certains membres du corps professoral qui supervisent le projet, les étudiants qui utiliseront la machine réalisée ainsi que les techniciens qui effectueron a supervision, l'entretien et la formation.

\section{$3.2 \quad$ Identification des besoins}

Afin d'établir la liste des besoins qui serviront à élaborer les bases de la machine $\mathrm{McGrO}$, un focus group a été réalisé avec tous les clients. La liste manuscrite des besoins énoncés par le client est disponible à l'annexe III. Le tableau 4 présente la liste des besoins conservés ${ }^{4}$.

\subsection{Analyse et classement des besoins}

En effectuant le regroupement des besoins du tableau 4 , on peut constituer le diagramme des affinités. Ce dernier permet d'avoir une vue globale des aspects qui preoccupent le client. Les besoins sont ainsi regroupes par afinite. Ces afinités devraient égalenent ressorir lors de lanalyse fonctionnelle puisquelles sont liés à natwe du projet. Tons les besoins ant été pondérs de 1 à 5 et identifies selon lenr classe. Une ponderation de 1 correspond a un besoin qui a peu dimportance relative

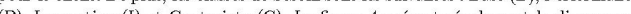
(P), In

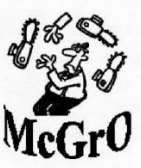

Thème: Suppont à atil allongr'

Pugh $\square$ Analyse paramétrique $\otimes$

Référence: $B, 13$ Niveau:

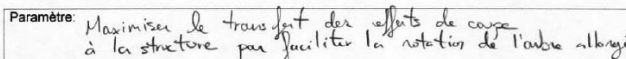
$\therefore 2 A$ or

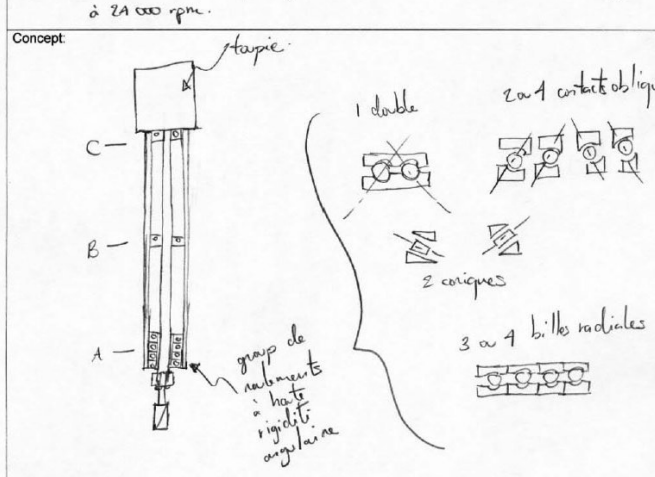
Evaluation: Atherition à la limitation de nigime ancicie an grapement

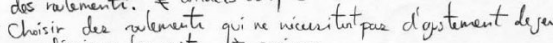
\$ Choisir der werments qui ne neer

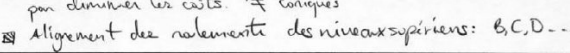

SHERBROOKE

B22

Date $(05,106102)$
Intiales du concepteur. JTP

\section{ANNEXE C - EXTRAITS DU RCD}

13

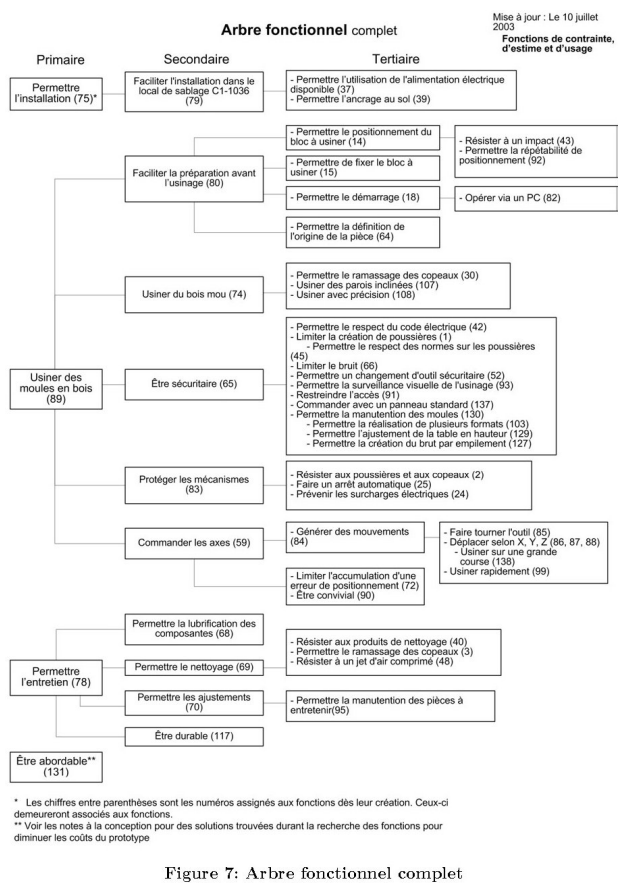

4.4.1 Géométrie 3D

Les parties mobiles de la machine qui permettront le déplacement de l'outil ont été nommées châssis mobiles. Le plus imposant d'entre-eux est le châssis mobile de l'axe X. Ce dernier supporte les antres châssis mobiles des axes Y et Z. La figure 25 montre ce châssis en mettant en évidence les pièces qui le composent Ce châssis a une forme de prisme triangulaire Le cadre à lui seul ne devrait pas dépasser une masse 65 k. Cette géométrie a été choisie pour limiter le poids maximiser la ridité cilite lase Cant le pont installé sur les glissières du châssis mobile de l'axe $\mathrm{X}$. Les dessins du châssis mobile de l'axe $\mathrm{X}$ sont disponibles à l'annexe III.

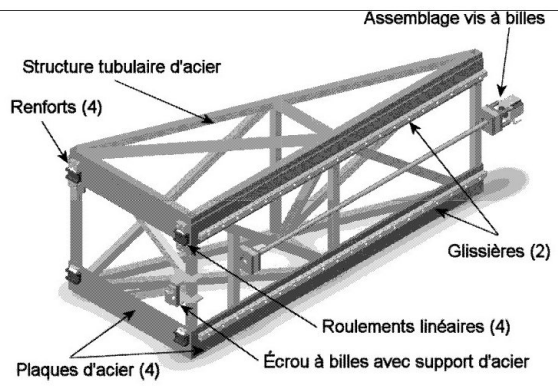

Figure 25-Châssis mobile de l'axe $\mathrm{X}$ 
4.4.2 Matériaux, technologies et fabrication

Cadre du châssis mobiles

Le châssis mobile de l'axe $\mathrm{x}$ est fait de tubes d'acier carré de 1 po de section $1 / 16$ po d'épais). Ces tubes sont coupés aux dimensions appropriées et assemblés ar soudage au MiG. Des aildsseurs sont integrés sur les laces du cadre tubulaire pur a d'o quatre plaques d acier assemblees sur les faces de ce chassis à angle droit sont d une épaisseur

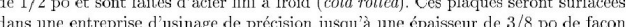
dans une entreprise d usinage de précision jusqu à une épaisseur de $3 / 8$ po de façon seront fixés les glissères et les roulements linéaires. Cette opération est indispensable puisque le retrait lors du soudage risque de déformer légèrement toutes les pièces du cadre. Pour les autres châssis mobiles, te boulonnage sera par conséquent priviligíé.

Alignement des glissières

Des guides de positionnement seront usinés lors de l'opération de surfaçage tel que montré à la figure 25 . Ceci a pour but d aligner la glissiere supérieure de l'axe Y ainsi que les roulements lineaires supérieurs de l'axe $\mathrm{X}$ à l'aide de cale. Les glissières et roulements linéaires inférieurs, pour leur part, seront positionnés avec le châssis mobile de l'axe Y sur les glissières.

Vis à billes

Le support de la vis à billes de l'axe Y sera soudé une fois assemblé et centré avec le châssis de l'axe Y mobile sur ses glissières. Le support de l'écrou à billes de l'axe $X$ sera soudé en le positionnant au mieux tel que montre sur la figure 25 et l'erreur vis a thlle $\mathrm{X}$.

Fixation des roulements linéaires

Des renforts en aluminium ${ }^{6}$ seront boulonnés au-dessus des roulements linéaires supérieurs de l'axe X pour ajouter quatre boulons supplémentaires de $1 / 4$ po de diamètre par roulement pour le support de ces derniers en tension. Les deux boulons 10-24 permettant de fixer ces roulements ne seront pas suffisants pour supporter sécuritairement le poids de l'assemblage des trois châssis mobiles en porte-à-faux. Les calculs de résistance de ces boulons sont présentés à la section 5.1.2.

6. L'aluminium 6061-T6 est utilisé pour les pièces de la machine à moins d'avis contraire

Annexe VI: Détails des calculs pour la validation du châssis mobile de l'axe $\mathrm{X}$

\section{A.1 Calculs par la méthode des éléments finis}

Toute la démarche qui suit s"inspire de la méthodologie enseignée dans le cours d'étude de cas IMC500 [PAN 02]

\section{A.1.0 Problématique}

Le châssis mobile de l'axe $\mathrm{x}$ doit être dimensionné afin de supporter les châssis mobiles des autres axes. Il doit évidemment être assez rigide pour que sa déformation ne crée pas un déplacement du bout de l'outil qui compromettrait la précision de l'ensemble de la machine. On veut donc evaluer la possibite d'uiliser un cadre en la machine McGrO. Les deux modèles de cadre envisagés sont montrés à la figure 69
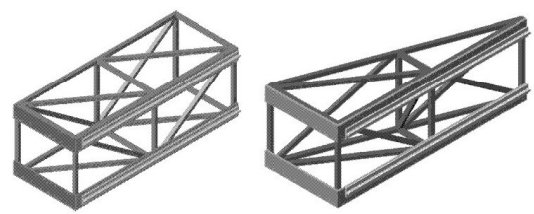

Figure 69 - Modèles de cadre étudiés pour le châssis mobile de l'axe $X$

A.1.1 Données du problème

Les forces appliquées sur le modèle sont les suivantes:

- Force de coupe de - $180 \mathrm{~N}$ appliquée au bout de l'outil selon l'axe X;

- Forces d'inerties générées par des accélérations de $10 \mathrm{~m} / \mathrm{s}^{2}$ pour les trois châssis mobiles $X, Y$ et $Z$ qui ont des masses respectives de 60,10 et $20 \mathrm{~kg}$ :

- Forces de gravité $\left(9.8 \mathrm{~m} / \mathrm{s}^{2}\right)$.

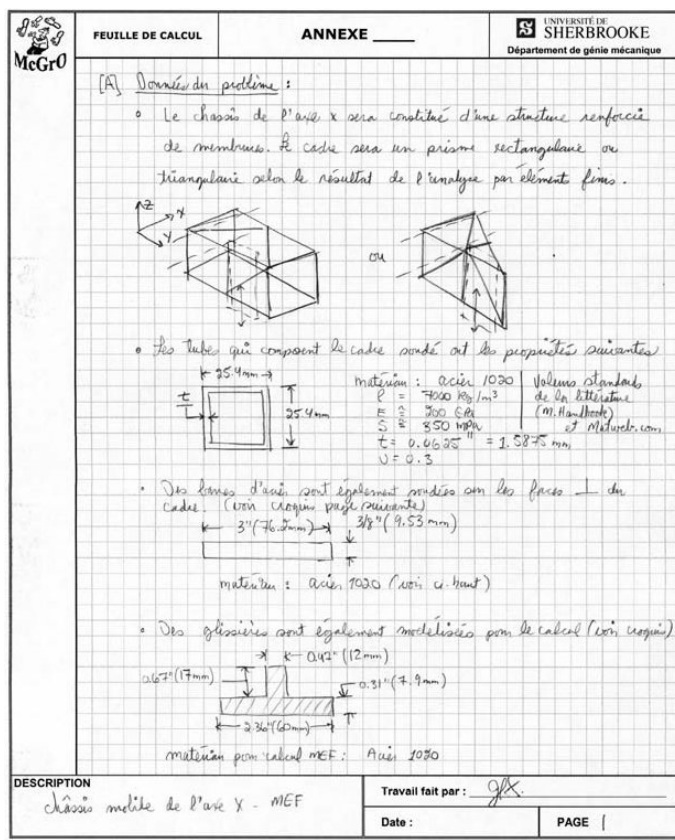

ANNEXE D - EXTRAITS DU RVE

5 Plan de validation expérimental au niveau des sous-systèmes

Voici maintenant la validation des aspects techniques de la machine McGrO. Étant donné le nombre important de spécifications d'ingénierie du CdCF, des tests de validation seront élaborés en les regroupant par sous-système ou affinité. Ces tests son présentés dans les pages qui suivent. Toutes les spécifications sont bien entendu exraites du CdCF de l'annexe 11.

5.1 Test 1 - sous-système déplacement linéaire

5.1.1 Objectifs

1. Vérifier si toutes les composantes entrant dans le déplacement des axes de la machine offrent un déplacement de l'outil répondant aux critères du CdCF. Les pièces de la, machine inclues dans ce test sont les suivantes:

- Glissières et roulements linéaires pour les trois axes;

- Transmission de mouvement linéaire: vis à billes, écrou et leur support métallique;

- Rallonge d'outil.

2. Calibrer les paramètres de fonctionnement de la machine $\mathrm{McGrO}$ (vitesse en déplacement rapide, avance et accélération des axes). Les paramètres doivent être utilisables pour toutes les formes géométriques. Ces paramètres pourront différés de ceux du CdCF évidemment si la machine ne répond pas aux spécification d'ingénierie associées à ces derniers.

5.1.2 Méthodologie (montage, instrumentation, etc.)

On commence tout d'abord par identifier les spécifications d'ingénierie qui s'appliquent au test 1 . Le tableau 5 montre certaines spécifications liées à ce sous-système de la machine qui peuvent être évaluées par de simples mesures. Les résultats de validation associés au tableau 5 sont d'ailleurs présentés dans la section 5.1.3et le tableau 6 montre les autres spécifications d'ingénierie que l'on souhaite valider grâce au test 1 . Pour ces dernières, une démarche plus spécifique est nécessaire.

La démarche du test 1 pour la validation des spécifications du tableau 6 est la suivante

1. Conception d'une pièce simple dans Solidworks contenant des surfaces en 3 dimensions (voir figure 15); 